Harry HX Xia, PhD, MD, Series Editor

\title{
Natural history of hepatitis-related hepatocellular carcinoma
}

\author{
David Yiu-Kuen But, Ching-Lung Lai, Man-Fung Yuen
}

David Yiu-Kuen But, Ching-Lung Lai, Man-Fung Yuen, Department of Medicine, The University of Hong Kong, Queen Mary Hospital, Hong Kong, China

Author contributions: But DYK wrote the paper; Lai CL and Yuen MF supervised and reviewed the paper.

Correspondence to: Man-Fung Yuen, Department of Medicine, the University of Hong Kong, Queen Mary Hospital, Pokfulam Road, Hong Kong, China.mfyuen@hkucc.hku.hk Telephone: +852-2-8553984 Fax: +852-2-8725828

Received: January 18, 2008 Revised: January 30, 2008

\begin{abstract}
Hepatocellular carcinoma (HCC) is an important cause of cancer death in the world. It has great regional differences in the pathology and epidemiology. The variation is greatly influenced by the aetiologies of the disease. Hepatitis B and C infection are the most important risk factors. HCC incidence rates are higher but in decreasing trend in developing countries. However, the figures in the developed countries are contrary. Successful hepatitis B virus (HBV) vaccination programs, better food hygiene, increased global hepatitis $\mathrm{C}$ virus (HCV) prevalence and population migration are the possible explanations. A number of clinical and pathogenic differences exist between HBV- and HCVrelated HCC. HBV infection leads to the development of HCC through direct and indirect pathways as it has the ability to integrate into the host genome affecting cellular signaling and growth control. HCV causes HCC mainly through indirect pathways: chronic inflammation, cell deaths and proliferation. As a result, HCC is almost exclusively found in cirrhotic HCV patients while HCC is sometimes found in $\mathrm{HBV}$ patients without significant liver cirrhosis. Due to the different severities of liver cirrhosis and HCC extent, therapeutic strategies from resection, liver transplantation to symptoms palliation are available. Poorly differentiated histology, lack of fibrous capsule, large tumour size, early vascular invasion and elevated serum levels of alpha fetoprotein (AFP) are the features for more aggressive disease. Combined with markers of liver reserve and performance status, accurate scoring systems and models have been developed to predict patients' survival and match best treatment option.
\end{abstract}

(C) 2008 WJG. All rights reserved.

Key words: Natural history; Hepatitis; Hepatocellular carcinoma
Peer reviewer: Miguel Carneiro De Moura, Professor, Department of Gastroenterology, Medical School of Lisbon, Av Prof Egas Moniz, 1649-028 Lisboa, Portugal

But DYK, Lai CL, Yuen MF. Natural history of hepatitisrelated hepatocellular carcinoma. World J Gastroenterol 2008; 14(11): 1652-1656 Available from: URL: http://www.wjgnet. com/1007-9327/14/1652.asp DOI: http://dx.doi.org/10.3748/ wjg. 14.1652

\section{INTRODUCTION}

Hepatocellular carcinoma (HCC) is the third most common cause of cancer death in the world. It results in 598000 deaths per year worldwide. Because of its poor prognosis, this number of deaths is almost the same as the number of cases being diagnosed each year $(626000)^{[1]}$. From a global perspective, the two most important risk factors for HCC are chronic hepatitis B and C infection. The geographic distribution patterns of HCC and hepatits $B$ virus (HBV) almost coincide with each other. The characteristics of patients with HCC are influenced by the etiology and the status of the underlying liver disease. The understanding of its natural history may influence the prognosis and choice of treatment.

\section{HISTORY OF HCC}

HCC was believed to be a rare disease in the early 1900 s. In Germany, Eggel found only 163 cases of HCC autopsies when he surveyed the world literature for HCC. He proposed an anatomical classification that HCC was classified as nodular, massive and diffuse ${ }^{[2]}$. However, it was found that the disease was more prevalent outside Europe. In 1911, Yamagiwa suggested a classification based on the origin of the cancer cell, hepatocellular and cholangiocellular, with the terminology of "hepatoma" and "cholangioma" respectively. After Berman's report of the extremely high HCC incidence among young Mozambican males in 1951, the interest in the studies of HCC increased. It has been shown that there is great regional difference in the pathology and epidemiology of the condition.

\section{DIFFERENT HCC PATTERNS}

HCCs in South Africa are mostly large sized and poorly 
differentiated with relatively healthy surrounding liver tissues. On the contrary, HCCs in non-African countries are relatively smaller in size and more differentiated with a background of cirrhotic liver. This can be the result of the difference in aetiologies. Aflatoxin B1, a mycotoxin produced by Aspergillus species, is believed to be a major carcinogenic factor in the South African population. Chronic hepatitis and the resultant cirrhosis are believed to be important risk factors in non-Africans. The distinct fibrolamellar type of HCC almost exclusively found in young Caucasian patients may be due to ethnic factor.

\section{HCC INCIDENCE}

Liver cancer incidence rate varies widely from 52.1 per 100000 in China to 5.1 per 100000 in Northern Europe. Developing countries contribute more than $80 \%$ of cases with China alone accounting for $55 \%{ }^{[1]}$. Other areas with high incidences are sub-Saharan Africa, eastern and southeastern Asia, and Melanesia. In the past decades, liver cancer incidence has decreased in some areas with high incidence in the past such as Shanghai, Singapore and Hong Kong. However, the opposite was reported in some of the countries in Europe, North America and Oceania. It is believed that effective $\mathrm{HBV}$ vaccination programmes and the better control of aflatoxin exposure in the high HCC incidence areas are contributing to the decreased HCC incidence while the increasing HCC incidence in some of the western countries is attributed to the increasing prevalence of hepatitis $\mathrm{C}$ virus (HCV) infection and immigration of people from countries with high endemicity for HBV infection.

\section{RISK FACTORS FOR HCC}

The main established risk factors for HCC development are chronic viral hepatitis $\mathrm{B}$ and $\mathrm{C}$ infection and aflatoxin B1. Liver cirrhosis per se may also lead to HCC development. Hepatits $\mathrm{C}$ infection probably causes HCC through the pathway of cirrhosis. Alcoholic liver disease, autoimmune liver diseases, primary hemochromatosis and Wilson's disease are also associated with the development of HCC. Overall, hepatitis B and C infections are causally associated with over $80 \%$ of HCC in the world ${ }^{[3]}$.

Worldwide, there are 400 million people infected with chronic hepatitis $B$, seventy five percent of whom are Asians. The natural history of chronic hepatitis B infection in Asian and African countries is different from that in the western world. In Asia and Africa, the majority of the people acquire the infection during the perinatal period or during very early childhood. There is a characteristically prolonged immune tolerance phase in the first few decades of life. The HBV viral loads are high and the liver transaminases are nearly normal. The lifetime risk of HCC in infected men is estimated to be $10 \%$ to $25 \%$ while the risk in women is somewhat lower $^{[3]}$. Case-control studies have shown that chronic HBV carriers have more than 100-fold increased risk of HCC compared with non-infected individuals. Furthermore, the risk of HCC development is related to the disease status of chronic hepatitis B infection. For example, the estimated annual risk of HCC in chronic carriers is $0.26 \%$ to $0.6 \%$. The risk increases to $1 \%$ in patients with active hepatitis ${ }^{[4]}$. It further increases to $2 \%$ to $3 \%$ in cirrhotic patients. Certain viral status also poses a higher risk of HCC. Studies in Taiwan showed that genotype $\mathrm{C}$ chronic hepatitis $\mathrm{B}$ infection has a more aggressive progression than genotype $\mathrm{B}$ in $\mathrm{HBeAg}$ positive patients ${ }^{[5]}$. Core promoter mutations (T1762/ A1764) are also found to be related to a more progressive disease $^{[6]}$. In the majority of HCC cases $(70 \%$ to $90 \%)$ there is underlying liver cirrhosis. However, HBV, being an oncogenic virus, can cause HCC in the absence of cirrhosis through the pathway of integration into the human genome.

\section{HBV CARCINOGENESIS}

As with many malignancies, the carcinogenesis of HCC is a multi-step process involving a number of different genetic alterations that ultimately lead to malignant transformation of the hepatocyte. It is postulated that HBV infection causes HCC via direct and indirect pathways. Continuous hepatocyte injury and regeneration in cirrhosis of the liver leads to increased liver cell turnover and hence accumulation of critical mutations in the host genome. This may result in genetic alterations, such as chromosomal rearrangements as well as activation of cellular oncogenes or inactivation of tumour suppressor genes. However a higher rate of chromosomal abnormalities is found in HBV-related HCC than those linked to other risk factors ${ }^{[7,8]}$. Alternative mechanisms in HBV carcinogenesis must also be involved. HBV belongs to the group of oncogenic viruses known as hepadna virus. It is able to integrate its DNA into the genome of the infected cell. Integrated HBV sequences have been observed in established hepatoma cell lines and in about $80 \%$ of human HBV related $\mathrm{HCCs}^{\left[{ }^{[0]}\right.}$. It is postulated that the HBV DNA integration may confer a selective growth advantage on target cells and leads to the onset of tumor progression. The integration sites are frequently detected in cellular genes involving cell signaling or growth control. Host chromosomal instability is also enhanced by HBV DNA integration. Large inverted duplications, deletions, amplification, chromosomal translocation have all been observed to be associated with HBV integration. In addition, the regulatory proteins $\mathrm{HBx}$ and the PreS2 activators can exert a tumor promoter-like function, resulting in positive selection of cells producing a functional regulatory protein.

\section{HCV CARCINOGENESIS}

$\mathrm{HCV}$ infection is a more important factor than $\mathrm{HBV}$ in the development of HCC in western countries. Markers of $\mathrm{HCV}$ infection are found in a higher proportion of HCC patients than that in most of the Asian countries; ranging from $44 \%$ to $66 \%$ in Italy, $27 \%$ to $58 \%$ in France, $60 \%$ to $75 \%$ in Spain ${ }^{[10]}$. Japan, unlike other Asian countries, also has a high proportion of HCC caused by HCV infection accounting for $80 \%$ to $90 \%$ of all the cases ${ }^{[10]}$. 
HCC risk increases to 17 -fold in $\mathrm{HCV}$-infected patients compared with $\mathrm{HCV}$-negative subjects ${ }^{[11]}$. The risk of $\mathrm{HCC}$ occurrence is different among all HCV patients. It is a function of the degree of liver fibrosis and the time of acquisition of the infection. The risk for cirrhotic HCV patients (F4) was the highest with $5.8 \%$ per year, compared to those who had less fibrosis (F1-3, 0.5\% to $2.6 \%)^{[12]}$.

Because of the absence of reverse transcription activity of the HCV RNA virus, its viral genome unlike HBV is not able to integrate into the genome of the infected cell. Therefore, HCV causes HCC via an indirect pathway by causing chronic inflammation, cell death, proliferation and cirrhosis. Accordingly, HCV-related HCCs are almost exclusively found in patients with cirrhosis. However, there are studies raising the possibility that HCV may also operate through other pathways in promoting malignant transformation of hepatocytes. HCCs without cirrhosis in HCV-infected patients, though rare, have been reported. The transforming potential of NS3 protein and core protein has been described.

\section{CLINICAL DIFFERENCES BETWEEN HBV- AND HCV-RELATED HCC}

Apart from the difference in the carcinogenic mechanisms, there are a number of clinical differences between HBVand HCV-related HCC. For reasons yet to be known there is a larger proportion of male $\mathrm{HBV}$ infected patients with HCC than the HCV counterpart. The male-tofemale ratio is higher in HBV-related HCC than in HCVrelated HCC (6.7:1 and 3.3:1 respectively in one study $)^{[13]}$. Studies consistently show that HCC develops 10 years earlier in HBV carriers than in HCV carriers, a difference possibly due to the earlier age at exposure to the causative agent ${ }^{[13,14]}$.

Due to the different mechanisms involved in carcinogenesis, their rate of HCC development is also different. In a Japanese study, 795 patients with viral or alcoholic cirrhosis were observed from 1974 to 1989. Two hundred and twenty five patients $(27.8 \%)$ developed HCC. The cumulative rates of HCC development in the $180 \mathrm{HBV}$ cirrhotic subgroup at 3, 5, 10 and 15 years after follow up were $7.2 \%, 14.2 \%, 27.2 \%$ and $27.2 \%$ respectively. At 9 years after the follow up, the rate of HCC development began to decrease, and the cumulative rate remained the same thereafter. The corresponding rates in HCV-related cirrhosis were $10.4 \%, 21.5 \%, 53.2 \%$ and $75.2 \%$ respectively in the $349 \mathrm{HCV}$ patients. The authors conclude that the cumulative rate of HCC occurrence has a linear relationship with time in HCV patients whereas it seems to plateau after 10 years for $\mathrm{HBV}$ patients ${ }^{[15,16]}$. However, according to an Italian study, the cumulative rates of HCC at 5, 10 and 15 years continue to increase with $6.5 \%, 23.4 \%$ and $31.9 \%$ for patients with HBV-related cirrhosis, and $4.6 \%$, $24 \%$ and $56.2 \%$ for patients with HCV-related cirrhosis, respectively ${ }^{[17]}$. At the time of HCC diagnosis, a higher proportion of $\mathrm{HCV}$ infected patients than HBV infected patients has advanced liver histology and has a higher Child Pugh's score ${ }^{[13]}$.

The HCC tumor size and the growth pattern also tend to be different between HBV- and HCV-related HCCs. In most patients with HCV-related HCC, the tumors are more likely to be solitary, smaller sized and encapsulated whereas HBV-related HCC are more commonly infiltrative and multinodular ${ }^{[18]}$. Extensive hepatic involvement and portal vein invasion by the tumor were found in $44 \%$ and $52 \%$ respectively in $95 \mathrm{HBsAg}$-positive HCC patients compared to $25 \%$ and $28 \%$ respectively in $370 \mathrm{HBsAg}$-seronegative HCC patients ${ }^{[19]}$.

\section{NATURAL HISTORY OF HCC}

The natural history of HCC depends on tumour growth characteristics as well as the underlying liver cirrhosis. The majority of hepatitis-related HCC develops in cirrhotic livers with a relatively poor liver reserve. Given a wide range of tumor doubling time, some patients may die from liver failure before the tumor grows to an advanced stage. Features of HCC shown to indicate more aggressive behaviour include poorly differentiated histology, lack of fibrous capsule, large tumour size, early vascular invasion and elevated serum levels of alpha fetoprotein (AFP). Tumor growth rates have a wide range of variability even among patients of the same region and regardless of disease stage. Doubling time ranges from $1 \mathrm{mo}$ to $19 \mathrm{mo}$ with a median of 4 to $6 \mathrm{mo}^{[20]}$.

Vascular supply of HCC is derived from the hepatic arterial network and the efferent vessels of portal origin have been described to create arterioportal or arteriovenous shunts which serve as a low resistance path for tumour thrombi fragments to survive and spread within the portal efferent network. In a large autopsy study from Sweden, $56 \%$ of HCC had evidence of vascular invasion but biliary tract involvement was observed in only $4 \%{ }^{[25]}$. In another autopsy study in Japan, vascular involvement was noted up to $82 \%$ and the likelihood of vascular involvement was related not only to tumor size but also the macroscopic types ${ }^{[26]}$. The frequency of portal vein invasion is higher than hepatic vein invasion ${ }^{[27]}$.

A retrospective analysis was performed in the United States of America on 347 HCC patients who received a metastatic workup including bone scans and computed tomography scans of the chest, abdomen, and pelvis. Clinical and tumor characteristics were evaluated as risk factors for metastasis by univariate and multivariate methods. One hundred forty-five patients had metastases: 72 had thoracic, 57 had abdominal, and 34 had bone metastases. Poor tumour differentiation, multilobular spread, and tumour size of $5 \mathrm{~cm}$ or more were the strongest predictors of metastatic disease by logistic regression analysis ${ }^{[28]}$. In a Japanese study, the relationship between HCC specimen staining for AFP, serum AFP levels and pathological findings were examined. The disease prognosis was studied with respect to the staining for AFP in excised tumors. The mean serum AFP level in patients with positive AFP staining was significantly higher than in those with negative AFP staining. No significant relationship was found between AFP positivity and tumor size, tumor encapsulation, degree of vascular invasion, or the histological differentiation grade of the tumor. However, patients with AFP-positive 
carcinomas had a poorer prognosis than those with AFPnegative carcinomas (5-year survival rate of AFP-positive and negative groups were $26.7 \%$ and $56.5 \%$, respectively) ${ }^{[29]}$.

\section{PREDICTION OF SURVIVAL}

The prognosis of HCC has been dismal because patients with HCC usually present late and have already developed advanced liver cirrhosis. Curative resection was seldomly possible. Therefore, the life expectancies of patients with newly diagnosed HCC were classically measured in terms of weeks to months with mortality to incidence ratio close to 1 . Due to the increased awareness of the disease and HCC surveillance programmes in some parts of the world, HCC can be diagnosed at its earlier phase permitting timely curative interventions. In a Hong Kong study ${ }^{[30]}$, the clinical features and disease outcome of 306 HCC patients were analysed. The characteristics of those present with symptoms and those with the disease diagnosed by screening were compared. One hundred and forty two patients with HCC diagnosed by regular screening have a significantly lower serum AFP level, smaller tumour size, less bilobar disease, less portal vein infiltration and less distant metastasis compared with the 164 symptomatic subjects. As a result a higher proportion of patients could undergo curative resection $(26.8 \%$ vs $7.9 \%)$.

Scores and models for predicting survival probability are invaluable to tailor suitable treatment options. The traditional TNM cancer staging system has limited applicability as it does not take into account the possible poor liver function in HCC patients. Eighty percent of HCC patients cannot be treated with surgery, so the tumour pathologic information needed in TMN cancer staging is not available. This further limits its prognostic use. Child-Pugh score on the other hand was designed to predict survival in liver cirrhosis without any component of HCC tumour morphology and consideration of disease extension. The first widely accepted staging system to predict survival for HCC which incorporates tumour biology and hepatic function was proposed by Okuda et al in $1985^{[31]}$. Tumour size, serum bilirubin, albumin and the presence of ascites are included in the calculation of Okuda staging. In a Hong Kong study of 106 untreated unresectable HCC patients performed in 2001, the median survival was $5.1 \mathrm{mo}$ in stage I, $2.7 \mathrm{mo}$ in stage $I$ and $1.0 \mathrm{mo}$ in stage III. These figures were remarkably similar to the original report by Okuda, in which the median survival of stages I, II and III disease was 8.3, 2, and 0.7 mo respectively. Among other staging systems, only Okuda staging was independently correlated with prognosis in the final multivariate model. As more treatment options become available, newer prognostic models are introduced. The Cancer of the Liver Italian Group Programme (CLIP) proposed a staging system which has been shown to be superior to that of the Okuda stage with a higher number of categories and greater discriminant ability. It reveals a subgroup of patients with an impressively more favourable prognosis who could be candidates for more aggressive therapeutic strategies. On the other hand, it also sorts out a subset of patients with a median survival long enough to be considered for clinical trials of palliative antineoplastic treatment. It includes Child-Pugh score, tumour size, presence of portal vein thrombosis, and serum AFP. Another survival model is the Barcelona Clinic Liver Group (BCLC) staging system which also effectively selects patients for aggressive treatments. It is constructed from several cohort studies and randomized controlled trials (RCTs) by the Barcelona group. This classification uses variables related to tumour stage, liver functional status, physical status, and cancer-related symptoms, and links the five stages described (0, A-D) with a treatment algorithm. The therapeutic approach configured into the BCLC staging system for patients with non-surgical HCC is based on the study by Llovet et al in which asymptomatic patients without evidence of disease extension (vascular invasion or metastasis) had significantly better survival compared to those with more advanced disease ${ }^{[28]}$. Such patients are classified as having intermediate HCC and may be better candidates for tumour ablative interventions or chemoembolization.

\section{HBV VACCINATION}

HBV vaccination has been shown to be effective to protect against HBV infection. The vaccination is recommended to high risk population groups such as health care workers, patients with immunocompromised states or with chronic liver diseases or with the need for regular blood product transfusion or hemodialysis, and neonates of HBV carrier mothers. Vertical and early horizontal transmission is the major route of $\mathrm{HBV}$ transmission in high prevalent areas. The transmission rate was estimated to be as high as $90 \%$ in $\mathrm{HBeAg}$ positive mothers. The use of $\mathrm{HBV}$ vaccine and hepatitis $B$ immunoglobulin (HBIG) can greatly reduce the infection rate of the neonates to $10 \%$ to $15 \%{ }^{[33]}$. The use of vaccination in all newborns can reduce the chance of infection acquired via other routes during early childhood and thus preventing the prolonged immunotolerant phase of this unique infection. By eliminating HBV seroprevalence in the population, HCC incidence should be greatly reduced in high prevalence areas. In Taiwan, a universal HBV vaccination programme was carried out in 1984. A study had shown that the HCC incidence in children was decreased since the introduction of the vaccination programme ${ }^{[34]}$. In that study, the author showed that the annual incidence of childhood liver cancer was reduced from 0.70 per 100000 to 0.36 per 100000 in the children born between 1981 to 1986 and 1990 to 1994 respectively. Therefore a similar decrease in the adult HCC incidence is expected in the future. Likewise, vaccination programmes in high endemic areas like Gambia, intermediate endemic areas like Italy had shown impressive reduction of the HBV seroprevalence. This may transform to a reduction in HCC incidence similar to the Taiwan study. However, the development of an effective vaccine has been hampered by the high genetic variability of HCV.

\section{CONCLUSION}

HCC is a complex neoplasm often occurring in a preneoplastic cirrhotic liver, and thus, survival and treatment options depend on variables of both HCC and cirrhosis. 
Among chronic viral hepatitis-related HCC, differences in carcinogenesis and clinical presentation exist between HBV and HCV patients. Survival models using tumour size, invasiveness, liver function, patient physical performance status can predict prognosis and guide treatments.

\section{REFERENCES}

1 Parkin DM, Bray F, Ferlay J, Pisani P. Global cancer statistics, 2002. CA Cancer J Clin 2005; 55: 74-108

2 Eggel H. Ueber das primare Carcinom der Leber. Beitr Pathol Anat 1910; 30: 506-604

3 McGlynn KA, London WT. Epidemiology and natural history of hepatocellular carcinoma. Best Pract Res Clin Gastroenterol 2005; 19: 3-23

4 Chu CM. Natural history of chronic hepatitis B virus infection in adults with emphasis on the occurrence of cirrhosis and hepatocellular carcinoma. J Gastroenterol Hepatol 2000; 15 Suppl: E25-E30

5 Yu MW, Yeh SH, Chen PJ, Liaw YF, Lin CL, Liu CJ, Shih WL, Kao JH, Chen DS, Chen CJ. Hepatitis B virus genotype and DNA level and hepatocellular carcinoma: a prospective study in men. J Natl Cancer Inst 2005; 97: 265-272

6 Liu CJ, Chen BF, Chen PJ, Lai MY, Huang WL, Kao JH, Chen DS. Role of hepatitis B viral load and basal core promoter mutation in hepatocellular carcinoma in hepatitis B carriers. $J$ Infect Dis 2006; 193: 1258-1265

7 Marchio A, Pineau P, Meddeb M, Terris B, Tiollais P, Bernheim A, Dejean A. Distinct chromosomal abnormality pattern in primary liver cancer of non-B, non-C patients. Oncogene 2000; 19: 3733-3738

8 Laurent-Puig P, Legoix P, Bluteau O, Belghiti J, Franco D, Binot F, Monges G, Thomas G, Bioulac-Sage P, ZucmanRossi J. Genetic alterations associated with hepatocellular carcinomas define distinct pathways of hepatocarcinogenesis. Gastroenterology 2001; 120: 1763-1773

9 Brechot C, Pourcel C, Louise A, Rain B, Tiollais P. Presence of integrated hepatitis B virus DNA sequences in cellular DNA of human hepatocellular carcinoma. Nature 1980; 286: 533-535

10 El-Serag HB, Rudolph KL. Hepatocellular carcinoma: epidemiology and molecular carcinogenesis. Gastroenterology 2007; 132: 2557-2576

11 Donato F, Tagger A, Gelatti U, Parrinello G, Boffetta P, Albertini A, Decarli A, Trevisi P, Ribero ML, Martelli C, Porru S, Nardi G. Alcohol and hepatocellular carcinoma: the effect of lifetime intake and hepatitis virus infections in men and women. Am J Epidemiol 2002; 155: 323-331

12 Kiyosawa $\mathbf{K}$. Trend of liver cirrhosis as precancerous lesions. Hepatol Res 2002; 24: 40-45

13 Shiratori Y, Shiina S, Imamura M, Kato N, Kanai F, Okudaira T, Teratani T, Tohgo G, Toda N, Ohashi M. Characteristic difference of hepatocellular carcinoma between hepatitis Band C- viral infection in Japan. Hepatology 1995; 22: 1027-1033

14 Fattovich G, Pantalena M, Zagni I, Realdi G, Schalm SW, Christensen E. Effect of hepatitis B and C virus infections on the natural history of compensated cirrhosis: a cohort study of 297 patients. Am J Gastroenterol 2002; 97: 2886-2895

15 Ikeda K, Saitoh S, Koida I, Arase Y, Tsubota A, Chayama K, Kumada H, Kawanishi M. A multivariate analysis of risk factors for hepatocellular carcinogenesis: a prospective observation of 795 patients with viral and alcoholic cirrhosis. Hepatology 1993; 18: $47-53$

16 Okuda $\mathbf{H}$. Hepatocellular carcinoma development in cirrhosis. Best Pract Res Clin Gastroenterol 2007; 21: 161-173

17 Gentilini P, Melani L, Riccardi D, Casini Raggi V, Romanelli
RG. Hepatocellular carcinoma and viral cirrhosis. Hepatology 1994; 20: 764-765

18 Okuda H, Obata H, Motoike Y, Hisamitsu T. Clinicopathological features of hepatocellular carcinoma-comparison of hepatitis B seropositive and seronegative patients. Hepatogastroenterology 1984; 31: 64-68

19 Shijo H, Okazaki M, Koganemaru F, Higashi M, Sakaguchi S, Okumura M. Influence of hepatitis B virus infection and age on mode of growth of hepatocellular carcinoma. Cancer 1991; 67: 2626-2632

20 Cottone M, Virdone R, Fusco G, Orlando A, Turri M, Caltagirone M, Maringhini A, Sciarrino E, Demma I, Nicoli N. Asymptomatic hepatocellular carcinoma in Child's A cirrhosis. A comparison of natural history and surgical treatment. Gastroenterology 1989; 96: 1566-1571

21 Ebara M, Ohto M, Shinagawa T, Sugiura N, Kimura K, Matsutani S, Morita M, Saisho H, Tsuchiya Y, Okuda K. Natural history of minute hepatocellular carcinoma smaller than three centimeters complicating cirrhosis. A study in 22 patients. Gastroenterology 1986; 90: 289-298

22 Barbara L, Benzi G, Gaiani S, Fusconi F, Zironi G, Siringo S, Rigamonti A, Barbara C, Grigioni W, Mazziotti A. Natural history of small untreated hepatocellular carcinoma in cirrhosis: a multivariate analysis of prognostic factors of tumor growth rate and patient survival. Hepatology 1992; 16: 132-137

23 Kubota K, Ina H, Okada Y, Irie T. Growth rate of primary single hepatocellular carcinoma: determining optimal screening interval with contrast enhanced computed tomography. Dig Dis Sci 2003; 48: 581-586

24 Liver Cancer Study Group of Japan. 14th report on liver cancer follow-up. Kyoto: Shinko Publication, 2000: 1996-1997

25 Kaczynski J, Hansson G, Wallerstedt S. Metastases in cases with hepatocellular carcinoma in relation to clinicopathologic features of the tumor. An autopsy study from a low endemic area. Acta Oncol 1995; 34: 43-48

26 Yuki K, Hirohashi S, Sakamoto M, Kanai T, Shimosato Y. Growth and spread of hepatocellular carcinoma. A review of 240 consecutive autopsy cases. Cancer 1990; 66: 2174-2179

27 Nakashima T, Kojiro M. Hepatocellular Carcinoma. An Atlas of its Pathology. Tokyo: Springer, 1987: 117-119

28 Si MS, Amersi F, Golish SR, Ortiz JA, Zaky J, Finklestein D, Busuttil RW, Imagawa DK. Prevalence of metastases in hepatocellular carcinoma: risk factors and impact on survival. Am Surg 2003; 69: 879-885

29 Izumi R, Shimizu K, Kiriyama M, Hashimoto T, Urade M, Yagi M, Mizukami Y, Nonomura A, Miyazaki I. Alpha-fetoprotein production by hepatocellular carcinoma is prognostic of poor patient survival. J Surg Oncol 1992; 49: 151-155

30 Yuen MF, Cheng CC, Lauder IJ, Lam SK, Ooi CG, Lai CL. Early detection of hepatocellular carcinoma increases the chance of treatment: Hong Kong experience. Hepatology 2000; 31: 330-335

31 Okuda K, Ohtsuki T, Obata H, Tomimatsu M, Okazaki N, Hasegawa H, Nakajima Y, Ohnishi K. Natural history of hepatocellular carcinoma and prognosis in relation to treatment. Study of 850 patients. Cancer 1985; 56: 918-928

32 Llovet JM, Bustamante J, Castells A, Vilana R, Ayuso Mdel C, Sala M, Bru C, Rodes J, Bruix J. Natural history of untreated nonsurgical hepatocellular carcinoma: rationale for the design and evaluation of therapeutic trials. Hepatology 1999; 29: 62-67

33 Chang MH. Hepatitis B virus infection. Semin Fetal Neonatal Med 2007; 12: 160-167

34 Chang MH, Chen CJ, Lai MS, Hsu HM, Wu TC, Kong MS, Liang DC, Shau WY, Chen DS. Universal hepatitis B vaccination in Taiwan and the incidence of hepatocellular carcinoma in children. Taiwan Childhood Hepatoma Study Group. N Engl J Med 1997; 336: 1855-1859

S- Editor Zhong XY L- Editor Alpini GD E- Editor Ma WH 